\title{
INFLUENCE OF PLASMON-PHONON COUPLING ON THE ELECTRON-IMPURITY INTERACTION IN THE FREE-CARRIER ABSORPTION*
}

\author{
W. Szuszkiewicz \\ Institute of Physics, Polish Academy of Sciences
}

Al. Lotników 32/46, 02-668 Warszawa, Poland

W. BARDYSZEWSKI

Institute of Theoretical Physics, Warsaw University, Hoża 69, 00-681 Warszawa, Poland

AND C. JULIEN

Laboratoire de Physique des Solides de l'Université Pierre et Marie Curie (URA 154)

4, Pl. Jussieu, 75252 Paris Cedex 05, France

An experimental and theoretical evidence that the electron-impurity scattering is modified in the presence of the electromagnetic field oscillating with the frequency corresponding to the optical phonon energy is given. The coupled plasmon-phonon mode related feature in the reflectivity spectrum can be used to determine the upper edge of the LO phonon frequency band in highly doped materials.

PACS numbers: $71.45 . \mathrm{Gm}, 78.30 . \mathrm{Fs}, 72.30 .+\mathrm{q}$

The infrared absorption in semiconductors is mainly caused by phonons and free carriers. In many cases these mechanisms can be treated separately, each of them giving its own contribution to the optical dielectric function. The simple Drude-Zener theory assuming frequency independent damping constants of free-carrier plasma and phonon excitations, satisfactorily describes most of the experimental reflectivity and transmission spectra in the infrared.

A deviation from this model can be expected when the light frequency approaches the optical phonon energy band. Strong mixing of the electron plasma and polar phonon modes leading to the collective motion of electrons and lattice ions causes a significant modification of the effective scattering of electrons by impurities [1]. This effect should reflect on the electron-impurity scattering rate giving a structure in the optical spectra at the energy corresponding to the upper edge of the LO phonon frequency band.

Most studies of the role of the dynamic screening in the free-carrier absorption were devoted to the question of plasmon excitation in the process of electron-impurity scattering (for the references see, e.g., [2]). In this paper we

*This work was supported by the State Committee for Scientific Research (Republic of Poland) grant No. 2P30219906. 
would like to consider the situation when the plasma frequency is much higher than LO phonon frequency. The effects of direct electron-phonon scattering in the temperature range considered in this paper is negligible. The dominant electron-impurity scattering rate is characterized by the frequency dependent inverse relaxation time

$$
1 / \tau(\omega)=\frac{\omega^{2} m^{*}}{e^{2} n} \operatorname{Re} \sigma(\omega),
$$

where $n$ and $m^{*}$ denote free electron concentration and effective mass, respectively. The real part of the high frequency conductivity is calculated from the expression valid when the phonon damping can be neglected [3, 4]:

$$
\begin{aligned}
& \operatorname{Re} \sigma(\omega)=-\frac{e^{2} N_{\mathrm{imp}}}{\omega^{3} m^{* 2}} \\
& \times \sum_{\mathbf{q}} q_{x}^{2}\left(\frac{4 \pi e^{2}}{q^{2} \varepsilon^{2}(q, 0)}\right)\left(\varepsilon_{\mathrm{L}}(q, \omega)-\varepsilon_{\mathrm{L}}(q, 0)+\varepsilon(q, 0)\right)^{2} \operatorname{Im}\left(\frac{1}{\varepsilon(q, \omega)}\right),
\end{aligned}
$$

where $\varepsilon(q, \omega)$ and $\varepsilon_{\mathrm{L}}(q, \omega)$ denote the total dielectric function and the lattice part of the dielectric function, the latter being defined by

$$
\varepsilon_{\mathrm{L}}(q, \omega)=\varepsilon_{\infty}\left(\frac{\omega_{\mathrm{LO}}^{2}-\omega^{2}}{\omega_{\text {TO }}^{2}-\omega^{2}}\right) .
$$

IIere $\omega_{\text {LO }}$ and $\omega_{\text {TO }}$ denote longitudinal and transverse optical phonon frequency and $\varepsilon_{\infty}$ is the high frequency dielectric constant.

The electron-impurity scattering rate is proportional to the loss function $\operatorname{Im}(1 / \varepsilon(q, \omega))$ which has a strong resonance at the $q$-dependent plasmon-phonon mode frequency. In this case the main contribution to the sum in Eq. (2) comes from the excitations with higher values of $q$, which are very weakly scattered by impurities. Consequently, inverse relaxation time is significantly reduced in this spectral region. The calculated dependence of $1 / \tau$ on the frequency $\omega$ in the vicinity of the phonon band excitations is presented in Fig. 1a. Apart from the phonon excitation band, the inverse relaxation time is relatively weakly frequency dependent. In our model calculations the optical phonon dispersion has been neglected (flat LO and TO phonon band approximation). In such a case the upper limit for LO excitations is equal to the LO phonon frequency at the $\Gamma$ point. The well pronounced minimum extending between $\omega_{T}$ and $\omega_{L O}$ frequency results from the modification of the impurity scattering. The edge type change of $1 / \tau$ at $\omega_{\text {LO }}$ gives a hope for experimental observation of the effect. The theoretical reflectivity curve obtained from our model presented in Fig. 1b possesses a small step at $\omega_{\text {LO }}$. This effect should be especially strong at high concentration of impurity centers, in particular under the condition of strong compensation. Obviously, the effect should be easier to observe in materials with higher ionicity where the plasmon-phonon coupling is more effective.

In order to verify the theoretical predictions the infrared reflectivity spectra were taken on the transition metal doped $\mathrm{HgSe}$ samples. High ionicity of this II-VI semiconducting compound is well known. The high doping level accompanied by the significant concentration of native defects in this material can cause a nonvanishing compensation. 

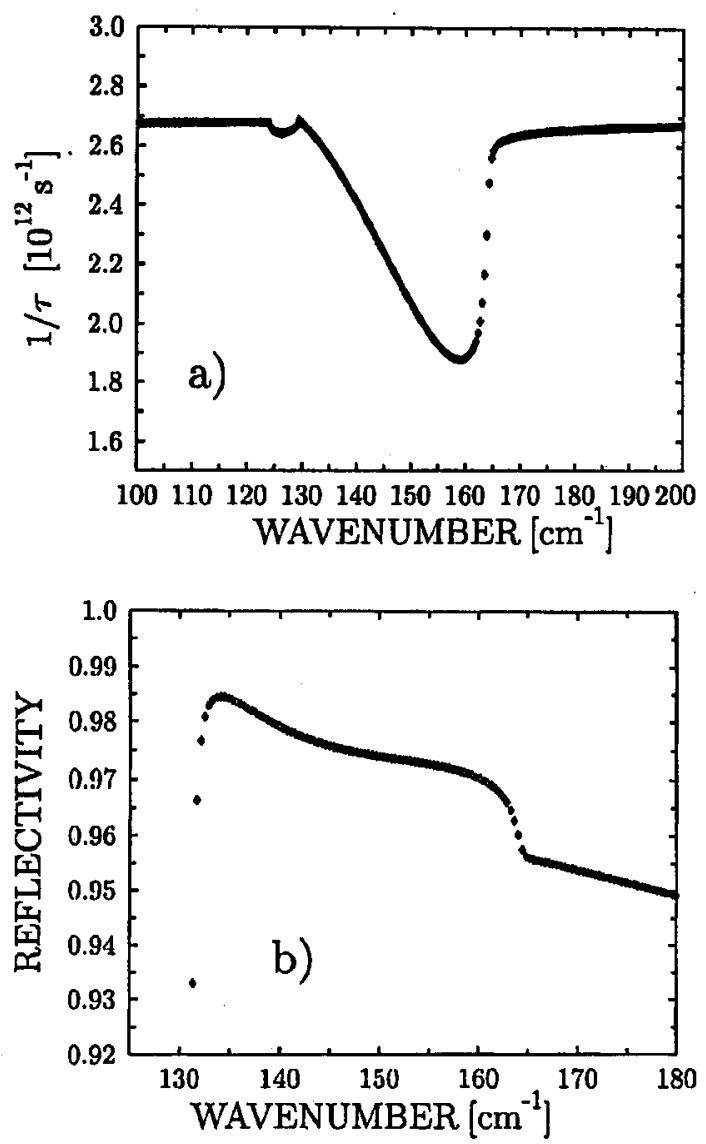

Fig. 1. (a) The theoretical dependence of $1 / \tau$ on the frequency $\omega$ in the vicinity of the phonon band excitation for parameters corresponding to HgSe. (b) Theoretical reflectivity versus frequency (part of the spectrum related to the effect discussed in text).

Single crystals of $\mathrm{Hg}_{1-x} \mathrm{Co}_{x}$ Se were grown by a modified Bridgman method. The Co concentration for the samples used in the optical experiments was determined by the electron microprobe and the energy dispersive X-ray fluorescence (EDXRF) analysis. The surface of the samples was prepared by the chemical etching in $\mathrm{Br} /$ methanol solution. The reflectivity was measured using the Fourier spectrometer in the spectral range from $100 \mathrm{~cm}^{-1}$ to $2000 \mathrm{~cm}^{-1}$ with the instrument resolution as high as $0.1 \mathrm{~cm}^{-1}$ in some particular cases. One millimeter thick samples were mounted on the cold finger of the helium cryostat.

A reasonable agreement with the Drude model of ac conductivity was achieved by using the free-carrier plasma frequency and damping as well as the oscillator strength and damping of TO phonon as fitting parameters. However, the experimental data obtained with high spectral resolution revealed a new feature close to the expected position of upper edge of the LO phonon frequency band, which 


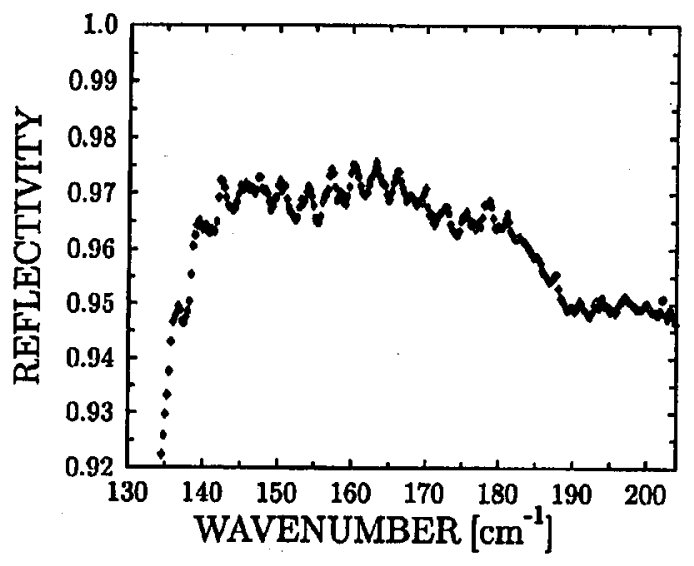

Fig. 2. Part of the experimental reflectivity spectrum taken for $\mathrm{Hg}_{0.995} \mathrm{Co}_{0.005}$ Se sample at helium temperature using Fourier spectrometer (instrument resolution equal to $0.5 \mathrm{~cm}^{-1}$ ). The small step predicted by the theoretical calculations occurs at slightly higher frequency due to the LO phonon dispersion.

may exceed the center of zone value by as much as $20 \mathrm{~cm}^{-1}$ [5]. In Fig. 2 a typical experimental reflectivity spectrum taken in the discussed phonon frequency range is presented. The slight step can be observed on the reflectivity curve at the frequency higher than that of the unscreened LO mode in $\mathrm{HgSe}$, equal to $173 \mathrm{~cm}^{-1}$, obtained from the Raman scattering measurements [6].

In conclusion, we would like to state that the scattering of electrons on ionized impurities in ionic crystals is strongly modified in the optical phonon frequency range. This effect is manifested by a small step in the reflectivity spectra at the position of the upper edge of the LO phonon frequency band. Thus a precise reflectivity measurements can provide the estimation of $\omega_{\mathrm{LO}}$ at the $L$ point of the Brillouin zone in highly doped ionic materials.

\section{Acknowledgment}

One of us (W.S.) would like to express his gratitude to Prof. M. Balkanski for his interest in this work and valuable remarks.

\section{References}

[1] M.E. Kim, A. Das, S.D. Senturia, Phys. Rev. B 18, 6890 (1978).

[2] W. Bardyszewski, W. Szuszkiewicz, Acta Phys. Pol. A 77, 273 (1990).

[3] S. Goettig, J. Phys. C, Solid State Phys. 17, 4443 (1984).

[4] W. Bardyszewski, W. Szuszkiewicz, Qian Dingrong, Zhang Jiaming, J. Cryst. Growth 101, 792 (1990).

[5] H. Kepa, T. Giebultowicz, B. Buras, B. Lebech, K. Clausen, Phys. Scr. 25, 807 (1982).

[6] K. Kumazaki, Phys. Status Solidi B 151, 353 (1989). 